\title{
Experiments and Computations on the Lift of Accelerating Flat Plates at Incidence
}

\author{
P.R.R.J. Stevens ${ }^{*}$, H. Babinsky ${ }^{\dagger}$ \\ Department of Engineering, University of Cambridge, CB2 1PZ, United Kingdom. \\ F. Manar ${ }^{\ddagger}$, P. Mancini ${ }^{\ddagger}$, A.R. Jones ${ }^{\S}$ \\ Department of Aerospace Engineering, University of Maryland, United States of America. \\ T. Nakata ${ }^{\text {II }}$, N. Phillips"l , R.J. Bomphrey ${ }^{* *}$
}

The Royal Veterinary College, University of London, United Kingdom.

\author{
A.C. Gozukara ${ }^{\dagger+}$ \\ MGEO Division, Aselsan Inc., Turkey. \\ K.O. Granlund
}

North Carolina State University, North Carolina, United States of America.

M.V. Ol ${ }^{x o}$

U.S. Air Force Research Laboratory, Wright-Patterson AFB, Ohio, United States of America.

This paper discusses the force history and flow topology of accelerating flat plate wings. The work is a collaborative effort to study fundamental, unsteady low Reynolds number flows. The motion kinematics are designed to be relevant to the Micro-Air Vehicle flight regime. A combination of experimental and computational techniques are used to obtain data for comparison. There is a striking correlation of lift history data and flow topology from both experimental and computational datasets. It is found that the Leading / Trailing Edge Vortex core separation during the initial part of a surge motion can be reasonably well approximated by $c \cdot \cos \alpha$ and the Leading/Trailing Edge Vortex relative advection velocity is estimated to be $0.5 \cdot U_{\infty}$. This Leading / Trailing Edge Vortex relative advection velocity is a useful measure of how quickly the Trailing Edge Vortex moves away from the Leading Edge Vortex, which can influence lift for accelerating flat plates at high incidence angles. 


\section{Nomenclature}

\begin{tabular}{|c|c|}
\hline$a$ & Non-dimensional distance over which wing accelerates \\
\hline$a_{s}$ & 'Eldredge' transition smoothing parameter \\
\hline$c$ & Wing chord, $\mathrm{m}$ \\
\hline$c_{l, c i r c}$ & 2D, circulatory lift coefficient \\
\hline$R$ & Resolution of LEV trajectory measurement, $[\mathrm{Hz}]$ \\
\hline$s$ & Distance of wing travel, $[\mathrm{m}]$ \\
\hline$s / c$ & Non-dimensional distance travelled in chord lengths \\
\hline$t$ & Time, $[\mathrm{s}]$ \\
\hline$t_{1}$ & Time at the start of surge, [s] \\
\hline$t_{2}$ & Time at the end of surge, [s] \\
\hline$u$ & Velocity in $\mathrm{x}$-direction, $\left[\mathrm{ms}^{-1}\right]$ \\
\hline$u_{L E V}$ & LEV advection velocity, $\left[\mathrm{ms}^{-1}\right]$ \\
\hline$u_{T E V}$ & TEV advection velocity, $\left[\mathrm{ms}^{-1}\right]$ \\
\hline$U_{\infty}$ & Reference velocity, $\left[\mathrm{ms}^{-1}\right]$ \\
\hline$x_{L E V}$ & $x$-position of LEV relative to LE of wing \\
\hline$x_{T E V}$ & $x$-position of TEV relative to TE of wing \\
\hline$\alpha$ & Angle of incidence, $\left[{ }^{\circ}\right]$ \\
\hline$\alpha_{0}$ & Maximum angle of incidence, $\left[{ }^{\circ}\right]$ \\
\hline$\Gamma$ & Circulation, $\left[\mathrm{m}^{2} \mathrm{~s}^{-1}\right]$ \\
\hline$\Gamma_{L E V}$ & LEV Circulation, $\left[\mathrm{m}^{2} \mathrm{~s}^{-1}\right]$ \\
\hline$\Gamma_{\infty}$ & Theoretical steady state circulation assuming attached flow, $\left[\mathrm{m}^{2} \mathrm{~s}^{-1}\right]$ \\
\hline$R$ & Aspect Ratio \\
\hline$L E$ & Leading Edge \\
\hline
\end{tabular}

\footnotetext{
${ }^{*}$ Research Associate, Cambridge University Engineering Department Aerodynamics Lab, Member AIAA, robbie.stevens@cantab.net

${ }^{\dagger}$ Professor of Aerodynamics, Cambridge University Engineering Department Aerodynamics Lab, Associate Fellow AIAA, hb@eng. cam. ac.uk

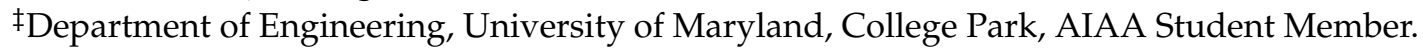

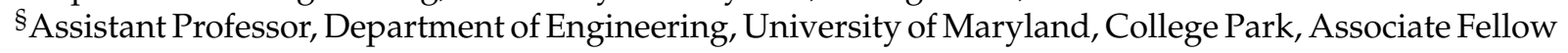
AIAA.

IIProject Assistant Professor, Graduate School of Engineering, Chiba University, Japan.

"Research Associate, Department of Comparative Biomedical Sciences, Royal Veterinary College, University of London.

${ }^{* *}$ Reader, Department of Comparative Biomedical Sciences, Royal Veterinary College, University of London.

${ }^{++}$Senior Engineer, MGEO Division, Aselsan Inc.

¥¥Assistant Professor, Department of Mechanical and Aerospace Engineering, Raleigh, AIAA Senior Member

${ }^{x o}$ Technical Advisor, Aero Configurations Branch, Aerospace Systems Directorate, Associate Fellow AIAA.
} 
LEV Leading Edge Vortex

LIC Line Integral Convolution

TE Trailing Edge

TEV Trailing Edge Vortex

\section{Introduction}

The unsteady lift generation of flapping wings is of interest for Micro-Air Vehicle (MAV) design and additionally to better understand natural fliers. A MAV is a small aerial vehicle which has classically been described as having a wingspan of $15 \mathrm{~cm}$ or less [1]. The MAV scale corresponds to the size and flight speed of small birds and larger insects and produces Reynolds numbers in the range of $10^{4}-10^{5}$ [2].

The mechanism of how flapping wings generate large stroke averaged lift coefficients (see for example Lentink and Dickinson [3]) was the motivation for NATO task group, AVT-149 [4]. The AVT-149 work lead to insight into how lift is connected to wing geometry, flapping kinematics and Reynolds number etc. To gain a better understanding of unsteady lift generation mechanisms, one approach is to investigate simplified but well controlled canonical cases. Dickinson and Götz [5] investigated the unsteady forces and flowfield for a wing translated in a stationary fluid for Reynolds numbers of $O 10^{3}$ and studied the Wagner effect and Dynamic Stall. Later Beckwith and Babinsky [6] conducted similar work but with a finite wing, finding that accelerating wings at high incidence can produce high lift coefficients. Ringuette et al. [7] performed experiments on impulsively started flat plates normal to the motion direction. Interestingly they described the drag force on the plate according to Batchelor's [8] formulation which depends on an impulse which is dependent upon the circulation of the LEV pair created.

Chen et al. [9] performed a computational study on a flat plate at high incidences at low Reynolds numbers (in the range of 10 - 200) with accelerations governed by a power law and found that the computed lift revealed an LEV-induced lift augmentation evident across all powers and angles of attack. Baik et al. [10] studied both pitching and plunging aerofoils for Reynolds numbers in the range 5000 - 20,000 and interestingly found that the Reynolds number has a small effect on aerodynamic force generation.

Despite the very informative studies conducted by other researchers, it is clear that there is a need for a more detailed investigation the force contributions for accelerating flat plate wings at high incidences. Past studies have also demonstrated the usefulness of comparisons between numerical and experimental results for similar kinematics and Reynolds numbers (see for example Bansmer and Radespiel [11] who investigated an aerofoil undergoing an acceleration in the direction of the freestream velocity at a Reynolds 
Number of 100,000).

The work described in this paper is the result of studies undertaken as part of the NATO RTO task group AVT-202, 'Advances in Fundamental Unsteady Low Reynolds Number Flows' [12]. This paper focusses on one particular aspect of the work, namely the insight gained from one of the canonical test cases defined by the task group. A typical flowfield for one of these simplified flapping motions features flow separation at the leading edge, a Leading Edge Vortex (LEV) and a starting or Trailing Edge Vortex (TEV). This observation inspired companion work within the AVT-202 task group on a simple low-order model [13], where the force is broken down into vortex lift and a non-circulatory, 'virtual mass' (inertial) force ${ }^{\mathrm{a}}$. The vortex lift is assumed to be caused by a LEV/TEV pair. The work contained herein forms the foundation for the companion work on the task group's low-order lift force model. The canonical surging wing case motion kinematics are outlined and the results are presented in terms of the aerodynamic forces, the flow topology and the strength and position of the Leading and Trailing Edge Vortices (LEV and TEV). Finally, we present a brief explanation of how the data contained herein contributes to the companion work on the aforementioned low-order model.

\section{A. Motion Kinematics}

All the case data discussed in this paper involve a flat plate wing with a rounded Leading Edge (LE), surging ${ }^{\mathrm{b}}$ over a streamwise distance of either 1 chord (1c, fast case) or 6 chords (6c, slow case). The wing (of Aspect Ratio $(R) 4$ for the canonical case) is accelerated (over $1 \mathrm{c}$ or $6 \mathrm{c}$ ) at a constant incidence of $45^{\circ}$ to a Reynolds number of 20,000 (canonical case) and continues to translate at a constant velocity thereafter. The canonical case is designed to be simplistically representative of MAV flight regime style kinematics. Figure 1 shows a breakdown of the motions that make up typical flapping stroke on a flying insect. The work described here focusses exclusively on the simple linear translation in the hope of gaining a greater appreciation of the underlying fundamental physics of flapping flight.

Variations of the wing Aspect Ratio $(R)$ and Reynolds number are highlighted where appropriate. The kinematics are represented simplistically in Figure 2.

Both the fast acceleration over 1c and slow acceleration over $6 \mathrm{c}$ cases have a constant acceleration, which produces a theoretical velocity variation, which is quadratic in space as shown in Figure 3. They show the non-dimensional velocity, $u / U_{\infty}$ plotted against 'advective time', s/c. The velocity is non-dimensionalised by the steady state freestream velocity. Advective time is the distance travelled by the wing, $s$ non-dimensionalised by the wing chord, $c$. While under acceleration, the surge velocity profile is described by the

a'Virtual' or 'Added' mass is an inertial reaction force which contributes to lift when a wing is accelerated in a fluid from rest and a mass of fluid is accelerated with the wing.

b'Surging' here refers to a constant acceleration from rest to a steady state velocity in a quiescent fluid. 

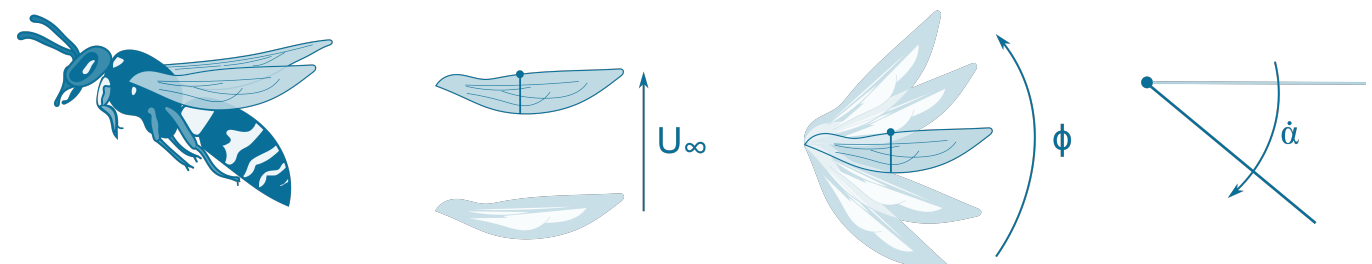

Figure 1: Simplification of flapping wing kinematics into a linear translation, revolution and rotation. Linear translation is the motion of interest in this work.

following expression:

$$
\frac{u}{U_{\infty}}=\frac{U_{\infty} t}{2 a c} \text { for } t<t_{2} \text { and } \frac{u}{U_{\infty}}=1 \text { for } t>2_{2}
$$

where $t$ is time and a is a non-dimensional distance over which the wing accelerates. $\mathrm{U}_{\infty}$ is the velocity which corresponds to the steady state freestream condition, whereas $u$ represents the instantaneous velocity so under steady state conditions this becomes $u=U_{\infty}$. The surge velocity profile may be described in terms of advective time by the following modified form of the above expression:

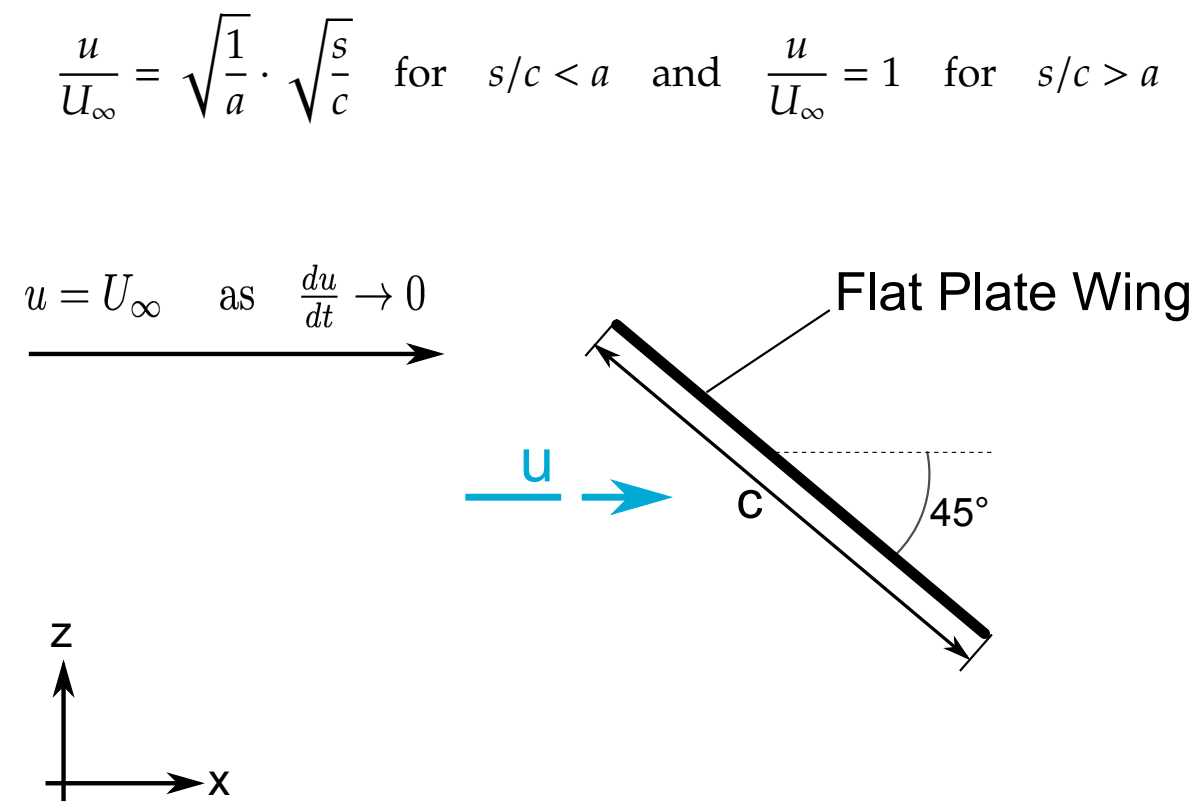

Figure 2: Canonical flat-plate wing surge kinematics. 


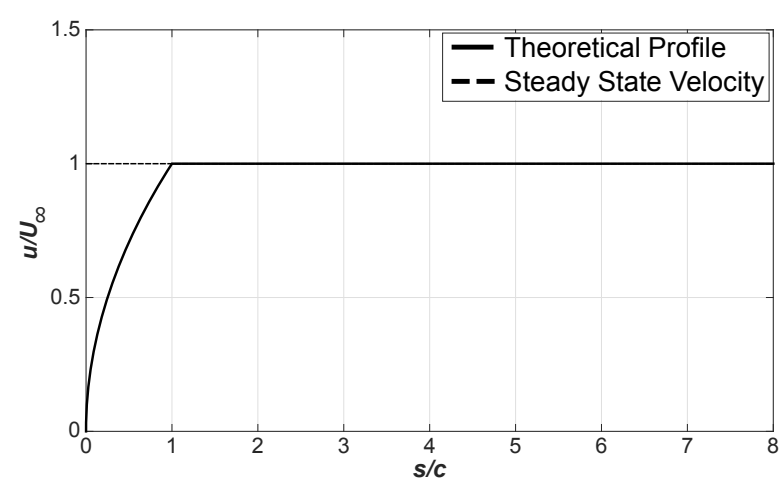

a) Fast surge (over $s / c=1)$.

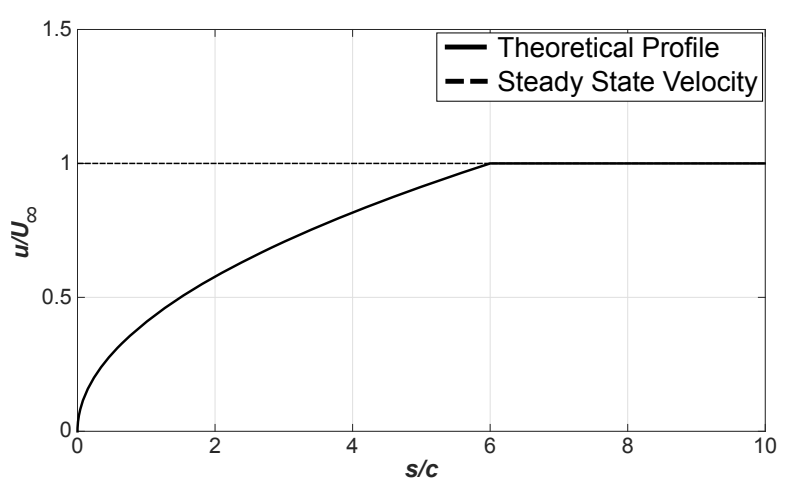

b) Slow surge (over $s / c=6)$.

Figure 3: Surge velocity profiles, steady state $\operatorname{Re}=20,000$.

\section{B. Link to Low-Order Model}

A key aspect of this paper is how experimental and computational data can be used to inform the development of a low-order model of the lift force [13]. It is identified there that the force may be separated into circulatory (viscous) and non-circulatory (inertial) contributions. The non-circulatory contribution is determined theoretically. It is shown however that the determination of circulatory lift requires knowledge of Leading Edge Vortex (LEV) and Trailing Edge Vortex (TEV) positions and velocities relative to the plate, and the circulation of the LEV according to the following expression:

$$
c_{l, \text { irc }}=-\frac{2}{U_{\infty}^{2} c}\left[\left(u_{L E V}-u_{T E V}\right) \Gamma_{L E V}+\left(x_{L E V}-x_{T E V}\right) \dot{\Gamma}_{L E V}\right]
$$

The values that need to be informed through experimental data may therefore be summarised as follows:

1. A LEV/TEV core separation distance, $\left(x_{L E V}-x_{T E V}\right)$.

2. A LEV/TEV, relative advection velocity, $\left(u_{L E V}-u_{T E V}\right)$.

3. The circulation of the LEV, $\left(\Gamma_{L E V}\right)$ and the rate of growth of LEV circulation $\left(\dot{\Gamma}_{L E V}\right)$.

The major assumption in attempting to determine the above values is that a distinct LEV and TEV form, which have equal but opposite strengths. Further, it is assumed that all the circulation which contributes to the lift may be considered to be that associated with the LEV and that bound circulation is negligible. In reality of course not all the circulation will necessarily be contained within the LEV but the implications of these assumptions seem to be small and are discussed in detail by Babinsky et al. [13]).

Figure 4 shows an adapted version of the comparison of force data alongside the prediction made by the low-order model of Babinsky et al. [13]. The authors state that the 
work did not aim to accurately predict lift but rather to identify various circulatory vs. non-circulatory forces, helping the understanding of the connection between unsteady force production and kinematics.

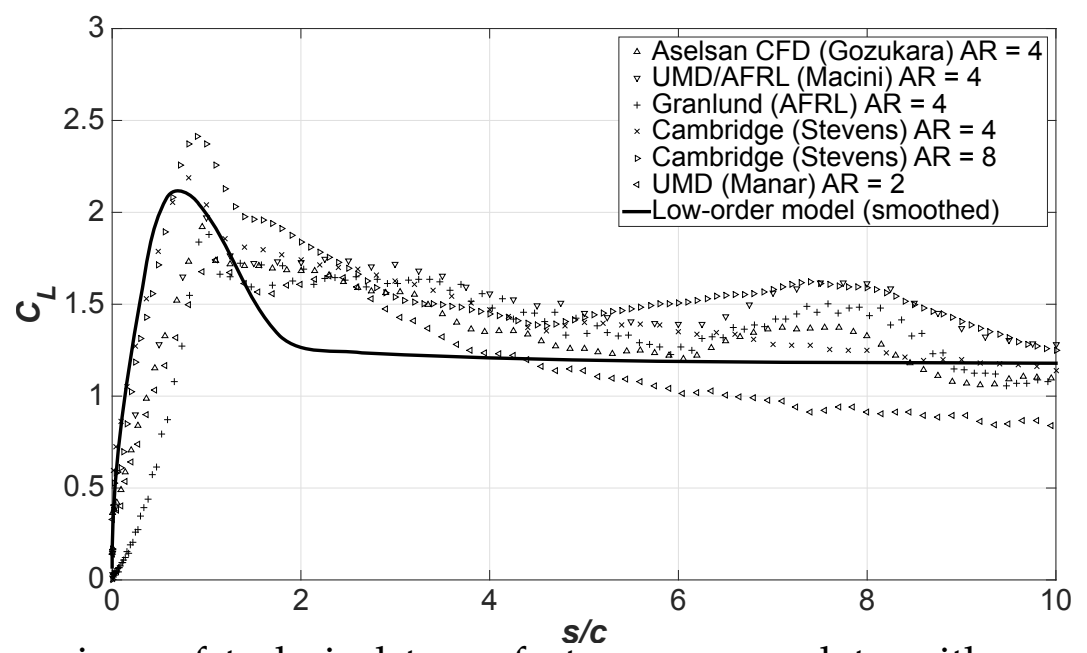

Figure 4: Comparison of technical team fast surge case data with surging theoretical model. Adapted from the companion work of Babinsky et al. [13].

The motivation of this paper is twofold: first to compare lift histories and flow topologies across a range of facilities and computational approaches and secondly to provide estimates of the aforementioned LEV and TEV parameters that are used in the prediction of the circulatory lift force in the companion work 'Low Order Modelling of Lift Forces for Unsteady Pitching and Surging Wings' [13].

\section{Methods}

This section overviews the facilities and methods used by the respective groups to obtain data. Data comes from two different experimental arrangements and two different computational approaches, giving a total of four independent data sources.

\section{A. Experiments}

1. University of Cambridge

The Cambridge University Engineering Department (P.R.R.J. Stevens and H. Babinsky) water towing tank facility is used for experiments. A schematic of the tow tank facility is shown in Figure 5. Experiments are conducted in water to take advantage of Reynolds scale effects. The tank has a $2 \mathrm{~m}$ long working section with clear side walls and floor for laser and camera optical access. The operational cross-section is $0.8 \mathrm{~m}^{2}$. A physical $R=2$ wing is used, which coupled with a surface skim plate to act as a symmetry plane, gives an effective $R$ of 4 . The wing has a chord length of $0.12 \mathrm{~m}$ and a thickness of $2.5 \%$. For 
the physical $R=2$ wing, blockage (at $\alpha=45^{\circ}$ ) is $2.5 \%$. Experiments are conducted at Reynolds numbers of 10,000 and 20,000.

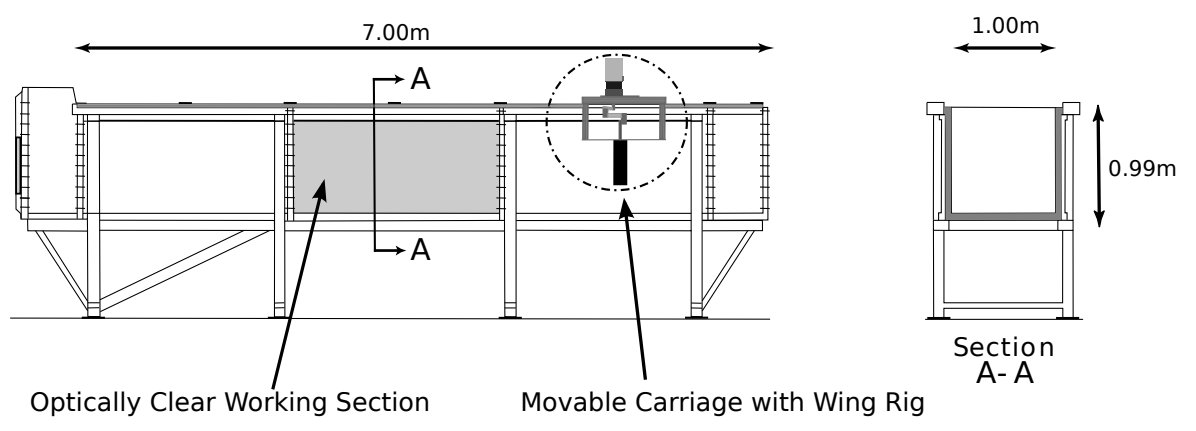

Figure 5: Tow tank facility schematic. (Adapted from Morgan [14])

There is a movable carriage mounted on top of the tank for towing the wing. The computer-controlled carriage can achieve a positional accuracy of $\pm 0.25 \%$. The acceleration ramp is smoothed using an Eldredge smoothing function (see Section II.B.1 for details). The carriage velocity is corrected for temperature variations greater than $1^{\circ} \mathrm{C}$, giving a maximum error in freestream Reynolds number of $1.25 \%$.

Lift forces are measured with a Flow Dynamics force balance of the strain gauge, load cell type. The two component balance has a range of $\pm 50 \mathrm{~N}$ and an accuracy within $0.01 \mathrm{~N}$. The balance is calibrated using known masses. Data is acquired at $1 \mathrm{kHz}$ and averaged over 10 runs. The data is smoothed using a moving-point average span of 100 data points with the endpoints treated by having progressively smaller span widths. To extract the pure fluid mechanical forces, it is necessary to subtract the inertial forces on the wing. This is done by performing the motion kinematics in air and assuming that the data acquired is representative of the inertial force exerted. This is valid if it is assumed that the aerodynamic forces in air are assumed small with respect to the inertial.

\section{U.S. Air Force Research Lab}

Data was taken in the United States Air Force Research Lab, Aerospace Systems Directorate's water tunnel, by K.O. Granlund and M.V. OL and separately by P. Mancini and A. Jones, thus producing two data sets. The tunnel is of the free-surface type, with test section $18^{\prime \prime}$ wide, 24 " high and 96 " long. The tunnel is fitted with a three degree of freedom motion system, consisting of three linear electric servomotors and a programmable controller. Two motors vertically oriented above the test section can be used to actuate pitch and plunge motions, while the third motor is used for the surge motions discussed in this paper. The surge motion is conducted, with the tunnel shut off and the two vertical motors set to place the model at $45^{\circ}$ incidence. Experiments are conducted at $\operatorname{Re}=20,000$. 


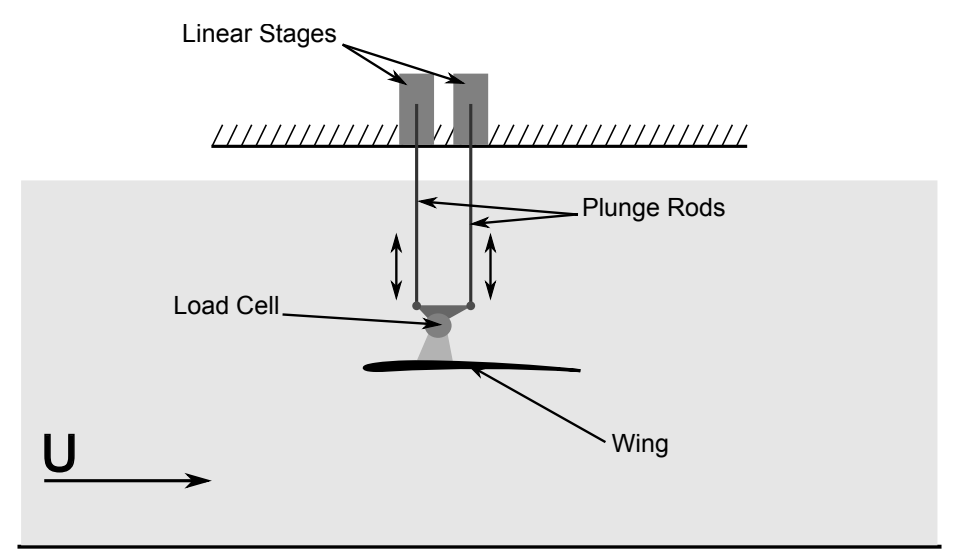

Figure 6: United States Air Force Research Lab, Aerospace Systems Directorate's water tunnel configuration.

The $R=4$ carbon fibre plate with $\mathrm{c}=0.0762 \mathrm{~m}$ and thickness $\sim 4 \%$ model is mounted horizontally in the test section, with a central support also housing the ATI Nano-25 6component force balance, which has a limiting range of $\pm 125 \mathrm{~N}$ and an accuracy of $0.02 \mathrm{~N}$. The plate is mounted offset from the load cell by a 2.25" 3D-printed plastic strut. Flow visualisation is conducted at 1c outboard from the wing's mid-span location.

Processing/filtering of force-data is discussed by Granlund et al. [15] with low-pass filtering ultimately limited by the system natural frequency, typically at $\sim 13 \mathrm{~Hz}$ in postprocessing, and at $18 \mathrm{~Hz}$ in the load-cell's native acquisition system, with sampling at $1 \mathrm{KHz}$. The system natural frequency was determined by a strike-test of the model.

\section{B. Computations}

\section{Royal Veterinary College}

CFD analysis is performed by the Royal Veterinary College (RVC) group (T. Nakata, N. Phillips and R.J. Bomphrey) with a versatile CFD-based dynamic flight simulator [16] that is based on three-dimensional, incompressible and unsteady Navier-Stokes equations. The wing is modelled by a single block $(101 \times 101 \times 101)$ that has a non-dimensional chord of 1 and uniform thickness of $1 \%$ with elliptical smoothing at all edges. The distance from the wing surface to the outer boundary is set to 18c. Translational velocity profiles were computed using the 'Eldredge function' [17], which is a method to smooth a surge profile. After Eldredge [18], the wing incidence, $\alpha(t)$ is described by the following function:

$$
\alpha(t)=\alpha_{0} \frac{G(t)}{G_{\max }}
$$

where, 


$$
\begin{gathered}
G(t)=\log \left[\frac{\cosh \left(a_{s} U_{\infty}\left(t-t_{1}\right) / c\right)}{\cosh \left(a_{s} U_{\infty}\left(t_{1}-t_{2}\right) / c\right)}\right]-a_{s} U_{\infty}\left(t_{1}-t_{2}\right) / c \\
G_{\text {max }}=2 a_{s} U_{\infty}\left(t_{2}-t_{1}\right) / c
\end{gathered}
$$

where, $\alpha_{0}$ is the maximum angle, $t_{1}$ is the time at the start of surge and $t_{2}$ is the time at the end of surge. The profiles were designed to reach constant velocity after 6 chords of travel. The RVC computations were performed using the prescribed canonical case geometry but with the Reynolds number set to 660 .

\section{Aselsan}

The Aselsan group (A.C. Gozukara) computed the fast surge case at a chord based Reynolds number of 10,000 using ANSYS Fluent 15.0. The computation was performed with an unstructured moving mesh composed of two zones; an outer grid surrounding an inner one, moving with the plate without going through a deformation. The outer zone is morphed and regenerated according to the predefined deformation rules to enable the inner zone to move. Mid-span cross-section grid slices are shown in Figure 7. The grid for RANS computations has 19426144 tetrahedral elements. Slightly smaller grids are used for laminar computations. The domain is $80 \mathrm{c}$ long, 40c wide and 40c high. The plate has a chord of $60 \mathrm{~mm}$, thickness of $\sim 3.3 \%$, and $R=4$. The computation is run as RANS with a $k$ - $\epsilon$ turbulence model. One chord of travel is divided into 500 equal time steps. A Re $=3600$ case has also been computed with flow solutions obtained by solving unsteady, laminar flow equations.

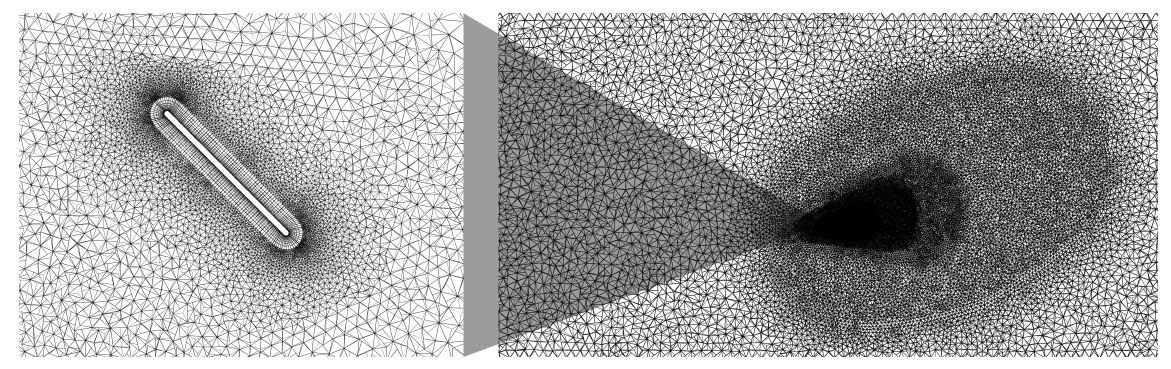

Figure 7: Cross-sectional view of Aselsan group's (A.C. Gozukara) unstructured grid.

\section{Discrepancies in Approach}

All groups are using a rectangular planform, $R=4$ wing for the canonical case, with a rounded LE and TE shape. Despite this effort to remain consistent, there are differences in the precise rounding of the LE and TE between all groups. It is also noted that the 
boundary conditions for experiments differ from each other as well. The Cambridge group uses a physical $R=2$ wing and a skim plate acting as an image plane, whereas the United States Air Force Research Lab uses a sting mounted wing with a physical $R=$ 4 wing. The difference in experimental boundary conditions could therefore have some effect on the flows experienced. For the Cambridge setup, there may be some influence of the the gap between the wing and the skim plate, so to mitigate this, the gap is set at $1 \mathrm{~mm}$ after the recommendation of Son et al. [19] who suggest that if a symmetry plane is used, the gap should be less than $2 \mathrm{~mm}$. For the United States Air Force Research Laboratory setup, the plastic strut will influence the flow in the central region of the wing. In practice however, it is found that generally such effects are small or difficult to discern. There are also some variations in the Reynolds number between cases, but it will be shown that there seems to be Reynolds number independence across the datasets. It is also recognised that despite all efforts to adhere closely to the acceleration velocity profile, across the methods, small differences in the transition region between the surge motion and constant velocity motion thereafter may affect force measurements but again it will be shown that the effect is relatively small.

\section{Results}

The canonical case is first discussed in terms of force history and flow topology. This allows a general appreciation of how specific flow features correlate to the forces at given instances. In the companion paper on the low-order model of the lift force, the LEV and TEV trajectories extracted from the data here are used to give position information and an approximation of their relative advection velocity.

\section{A. Force History}

Figure 8 compares lift coefficient histories for the fast and slow surge cases respectively. The lift is plotted against an advective timescale, $s / c^{c}$ It is immediately recognised that there is good agreement between different datasets. Considering the fast case of Figure $8 \mathrm{a}$, at first, there is an initial spike in lift, followed by a drop. The same trends are captured by all datasets astonishingly well, namely, the initial peak in lift, followed by a short, sharp drop, then a slow decay in lift before (with the exception of the data of Stevens) a second maximum in lift and finally an asymptotic decay to a steady state lift coefficient. Consider Figure 8c, which shows a zoom of the early portion of the lift history. The peak lift coefficient occurs towards the end of the first chord of travel within $15 \%$ of $s / c=1$ for all cases. The rates of growth, up to the first lift maximum are also quite similar in trend,

\footnotetext{
${ }^{\mathrm{c}}$ The advective time $s / c$ as used here, corresponds to the non-dimensional distance travelled.
} 
with the data having an approximately linear increase.

There are some subtle differences in how the lift increases up to the first maximum, with the data of Stevens showing a higher lift while surging and a peak lift that is $10 \%$ greater than any other, whereas the data of Granlund has a peak lift that is $17 \%$ lower than any other. There is also a very noticeable difference in the initial value of lift at $s / c=0$ for all cases, which varies from $c_{l} \approx 0.2$ (Mancini) to $c_{l} \approx 0.6$ (Stevens). Some of the differences which are observed may be accounted for by the type of data filtering and smoothing applied by each group. Returning to Figure 8 a, after the first maximum, there is initially a small but nonetheless, sharp drop in lift, this is followed by a slow decay which persists until approximately $s / c=6$. The datasets of Gozukara, Mancini and Granlund then all show a similar second maximum in lift around $s / c=7.5$. Interestingly, this feature is apparent in the data of Stevens, although there is a smaller, less distinct second maximum a little earlier at around $s / c=6$. The data of Stevens however shows that it takes long advective timescales for a steady state condition to be approached (around 20s/c in this case).

The experimental datasets generally show good agreement with the single CFD dataset of Gozukara, giving confidence in both the accuracy of the experimental datasets and the quality of the CFD. A typical deviation from the mean at $s / c=5$ for example is approximately $\pm 10 \%$.

The slow surge lift history of Figure $8 \mathrm{~b}$ shows a rise in lift, peaking at approximately $s / c=6$. There is an absence of a secondary rise in forces. The lift trace of Stevens shows a flattening of the lift coefficient around $s / c=15$. The value of the asymptotic lift coefficient here is approximately $25 \%$ of that observed where the curve flattens in the fast surge case, suggesting that this is not yet the true steady state lift coefficient. It may be that a much longer advective timescale is required to study this phenomenon.

\section{B. Flow Topology}

The discussion in this section is qualitative, with a quantitative investigation of the vortex dynamics and strength to follow in subsequent sections.

Figures 9 and 10 show the comparison of flow visualisation with vorticity fields with vectors superimposed from PIV and CFD data for the fast surge case. All figure dimensions are non-dimensionalised by the wing chord, $c$, clockwise fluid rotation is coloured blue with anti-clockwise rotation coloured red.

The data of Figure 9 are arranged to show the comparison of the initial development of the flow and the formation of the LEV, followed by the breakup of the LEV. There is no TEV structure present within the limited field of view for PIV data but this is clearly visible in the flow visualisation and CFD data for $s / c=0.5$ and 1.5. A distinct LEV structure has already formed at $s / c=0.5$. In the PIV data, a shear layer emanating from the TE is also 


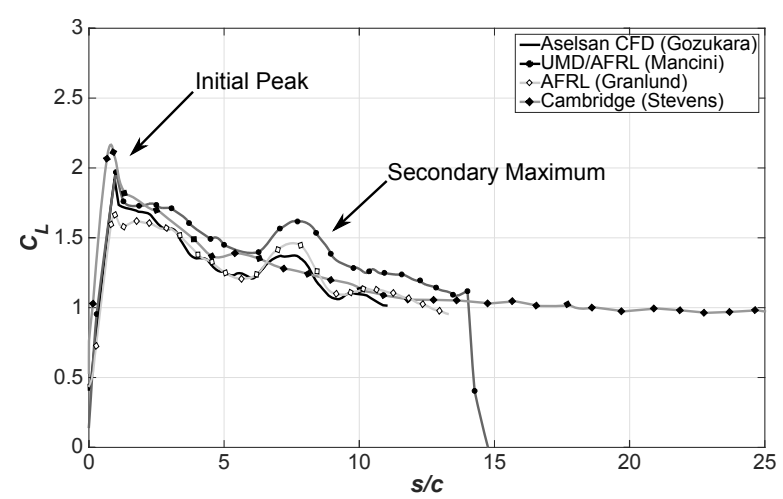

a) Fast surge lift coefficient history.

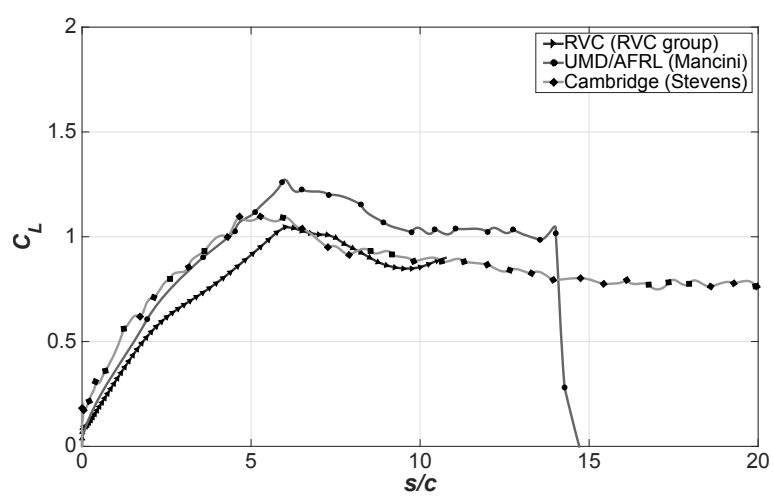

b) Slow surge lift coefficient history.

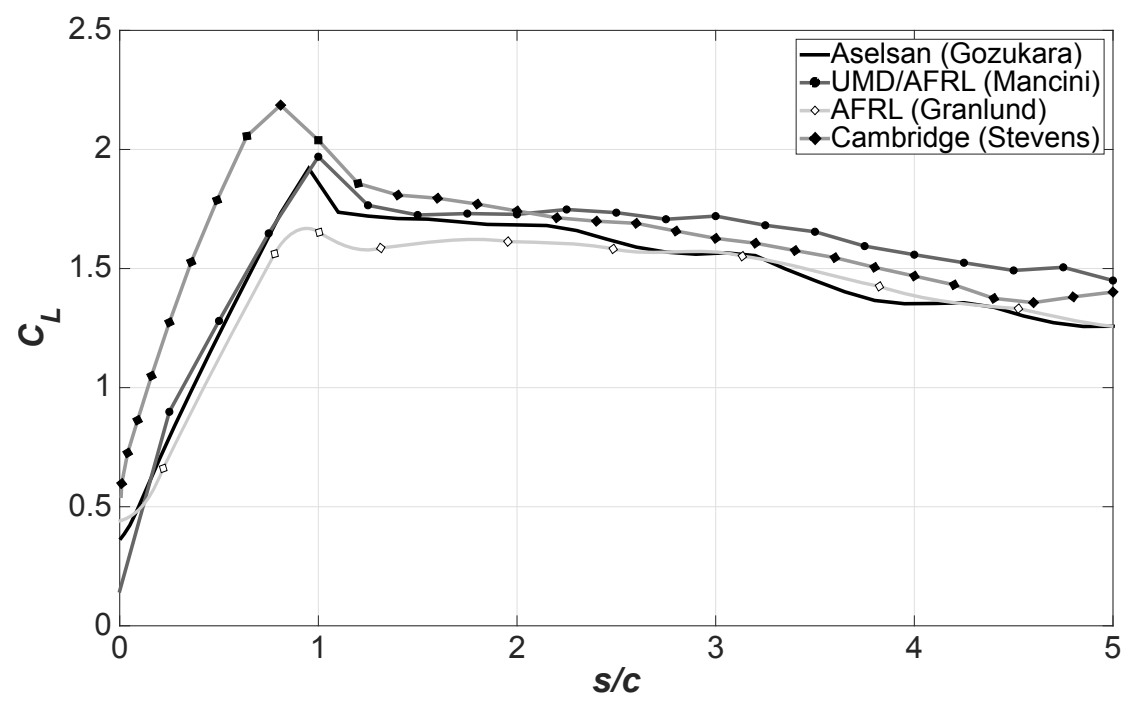

c) Zoom of lift history for early $s / c$ for the fast surge case..

Figure 8: Lift coefficient histories for fast and slow surge cases respectively.

visible, even though the TEV is not. By $s / c=1.5$, the LEV core has grown and advected aft of the LE of the wing in $x$, with hardly any change in the $y$-position. Additionally it is noted that the LEV has moved downstream from the LE less than the $s / c=1$ movement of the plate and hence is considered still somewhat 'attached'. At $s / c=2.5$, the LEV core has advected further aft, with a noticeable dispersion of the LEV beginning to occur. The TEV seen initially is still visible in the flow visualisation but in the CFD data it has now advected out of the field of view. Closer inspection of the flowfields indicates some variance in the LEV core position between the respective techniques although the general topology is remarkably similar.

Figure 10 shows the flow development for $s / c \geq 3.0$. The PIV shows a marked loss of LEV coherency by $s / c=3.0$, which is in contrast to the flow visualisation and CFD, where the LEV is still reasonably well defined. For all three methods, the flow appears stalled at 
$s / c=5.0$, although there is some new recirculating flow close to the LE, this is not coherent like the LEV previously observed. At $s / c=8.0$, there is no flow visualisation available but comparison of the PIV and CFD shows that a body of slowly recirculating fluid has formed above the wing, which is unusual and may be a streamline pseudo-reattachment of the flow. This pseudo-reattachment likely explains the presence of the second lift maximum, which was noticed to occur around $s / c=7.5$.

Figure 11 compares flow visualisation and PIV data $(\operatorname{Re}=20,000)$ in the first and second columns respectively with CFD data $(\operatorname{Re}=660)$ in the third and fourth columns for the slow surge case. Once more, all dimensions are non-dimensionalised by the wing chord, c. Data is again presented with clockwise fluid rotation coloured blue with anti-clockwise rotation coloured red. The CFD in the third column shows vorticity with velocity vectors superimposed for a direct comparison with PIV data. In addition Figure 11 also shows images generated using the Line Integral Convolution (LIC) technique [20] to visualise the CFD data.

Initial cross-examination of the flow visualisation, PIV and CFD data suggests that there is some agreement in the general nature of the flow structures observed.

Turning in detail to the flow visualisation in the left hand column of Figure 11, there is initially at $s / c=1.5$, a LEV structure visible and this is also reflected in the PIV and CFD data. By $s / c=3$, there is an absence of a clear LEV structure. Upon closer inspection of the vector field, there is a clockwise rotational tendency above the wing, however the vorticity colouring suggests that this rotation is weak. The CFD data does however seem to show a more distinct LEV. A direct comparison of the PIV flowfields may be made with the CFD flowfields of the third column. The CFD vectors show more distinct vortical structures and these are made very apparent by the LIC processing in the right hand column. In contrast, the flow visualisation and PIV data suggests that such structures are weak if they exist at all and the flow is more akin to separation. There may be some Reynolds number effect here but this is not strongly reflected in the force history.

\section{LEV and TEV Position}

The previous analysis of the flow topology has shown that LEV structures are particularly noticeable in the fast surge case, where they slowly advect away from the wing as it continues to move in translation. Here, vortex trajectories are tracked relative to the LE for the LEV and Trailing Edge (TE) for the TEV respectively ${ }^{\mathrm{d}}$. The position of the individual LEV and TEV in space enables their core separation, $\left(x_{L E V}-x_{T E V}\right)$ to be estimated and this in turn allows their relative advection velocity $\left(u_{L E V}-u_{T E V}\right)$ to be determined. These

\footnotetext{
${ }^{\mathrm{d}}$ Flowfields with the TEV visible are not shown in this paper but positional data was extracted from PIV datasets.
} 


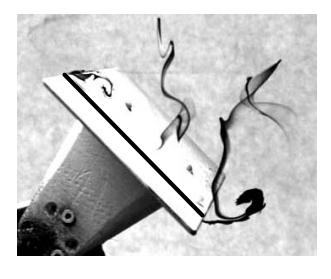

a) $s / c=0.5$ (Flow Vis)

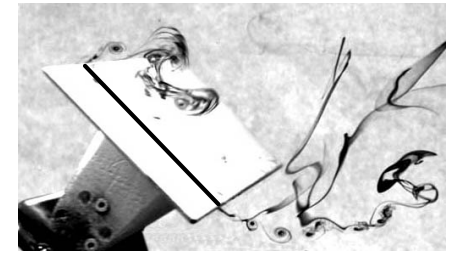

d) $s / c=1.5$ (Flow Vis)

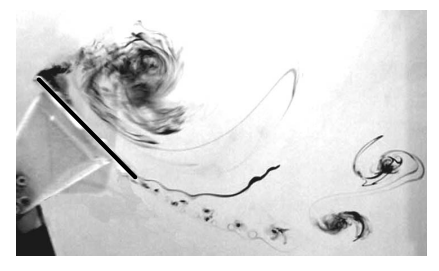

g) $s / c=2.5$ (Flow Vis)

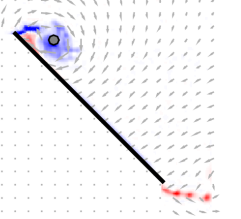

b) $s / c=0.5(\mathrm{PIV})$

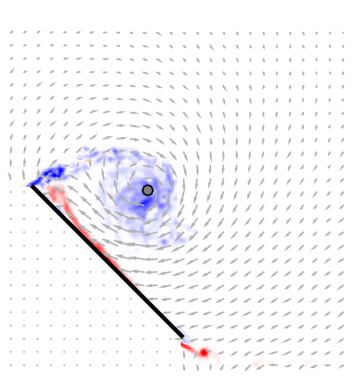

e) $s / c=1.5$ (PIV)

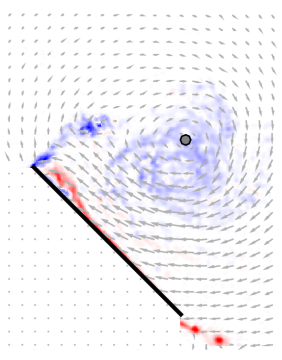

h) $s / c=2.5$ (PIV)

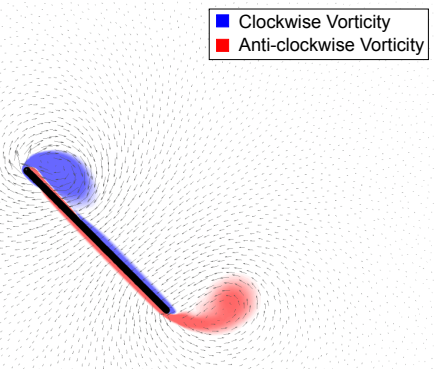

c) $s / c=0.5$ (CFD)

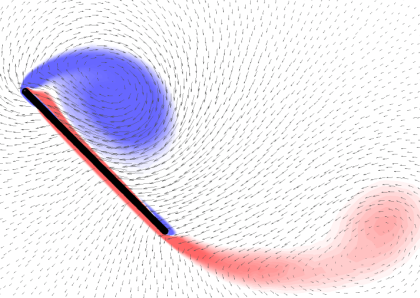

f) $s / c=1.5$ (CFD)

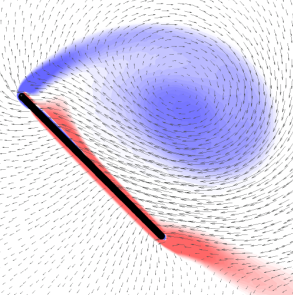

i) $s / c=2.5$ (CFD)

Figure 9: Comparison of fast surge flow fields from AFRL dye flow visualisation in the left hand column, UMD/AFRL PIV data showing vorticity field with vectors superimposed in the middle column and Aselsan CFD vorticity field with vectors superimposed in the right hand column for early advective times. 


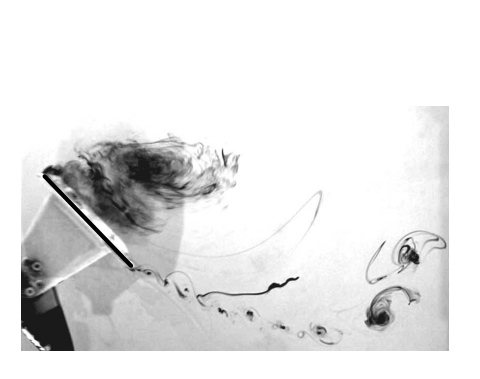

a) $s / c=3$ (Flow Vis)

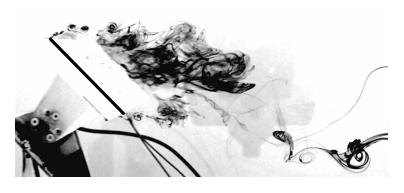

d) $s / c=5$ (Flow Vis)

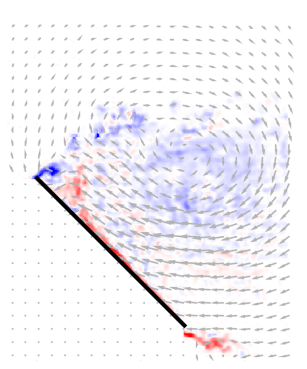

b) $s / c=3$ (PIV)

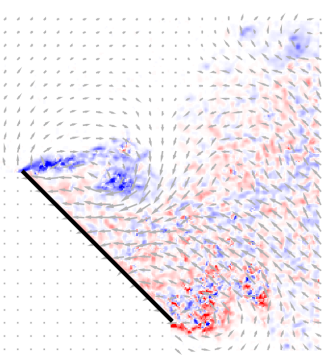

e) $s / c=5$ (PIV)

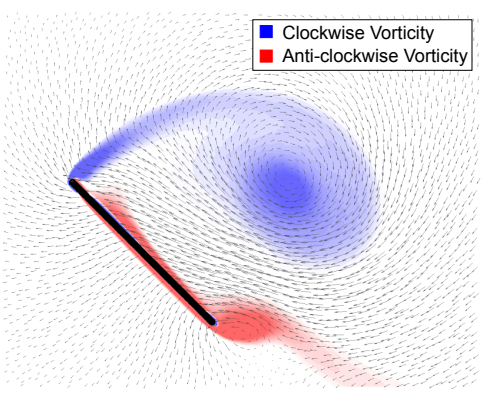

c) $s / c=3(\mathrm{CFD})$

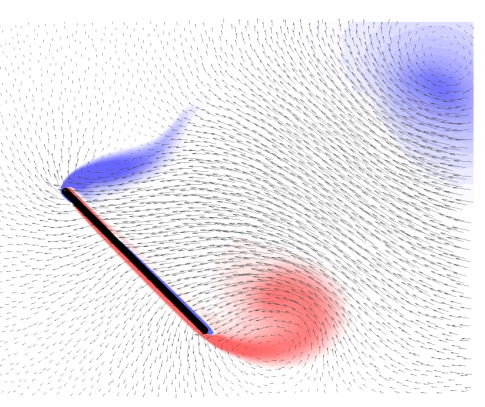

f) $s / c=5$ (CFD)

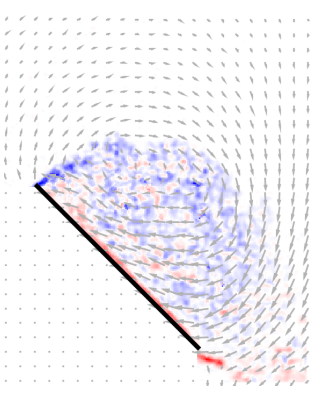

g) $s / c=8$ (PIV)

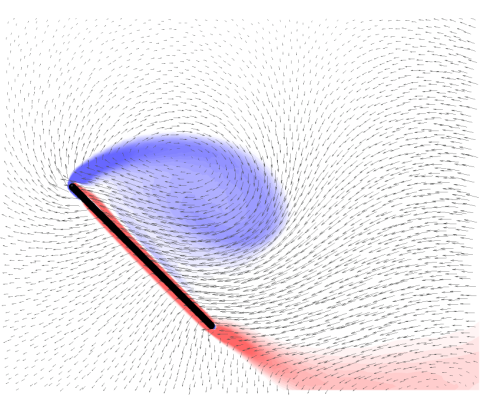

h) $s / c=8$ (CFD)

Figure 10: Comparison of fast surge flow fields from AFRL dye flow visualisation in the left hand column, UMD/AFRL PIV data showing vorticity field with vectors superimposed in the middle column and Aselsan CFD vorticity field with vectors superimposed in the right hand column for later advective times. 


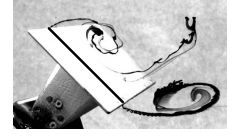

a) $s / c=1.5$ (Flow Vis)

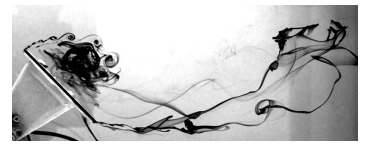

e) $s / c=3$ (Flow Vis)

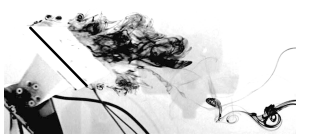

i) $s / c=5$ (Flow Vis)

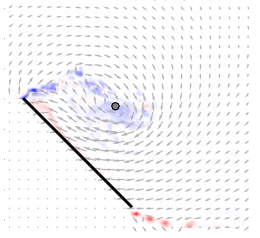

b) $s / c=1.5$ (PIV)

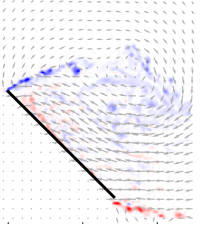

f) $s / c=3(\mathrm{PIV})$

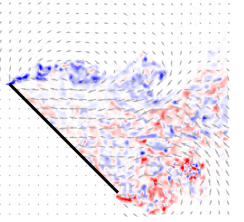

j) $s / c=5$ (PIV)

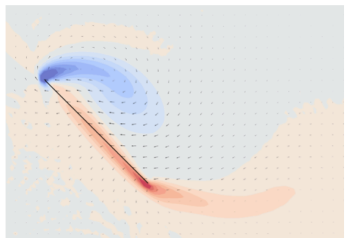

c) $s / c=1.5$ (CFD)

d) $s / c=1.5$ (CFD)

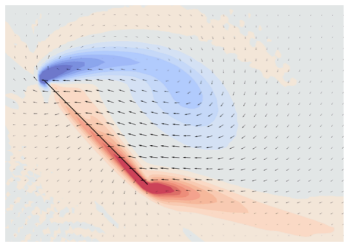

g) $s / c=3$ (CFD)

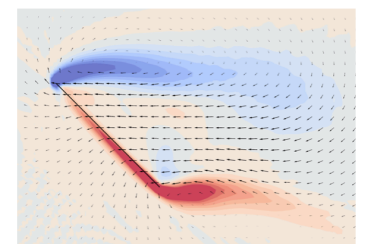

k) $s / c=5$ (CFD)

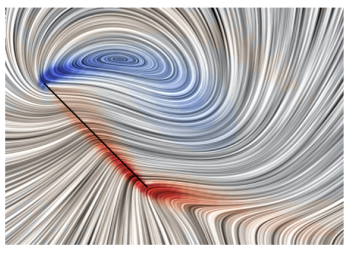

h) $s / c=3$ (CFD)

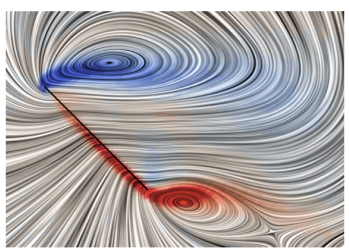

1) $s / c=5$ (CFD)

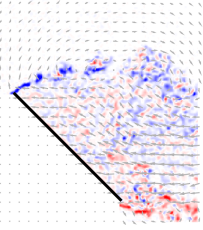

m) $s / c=8$ (PIV)

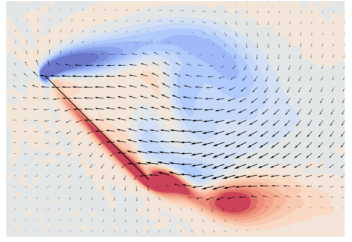

n) $s / c=8$ (CFD)

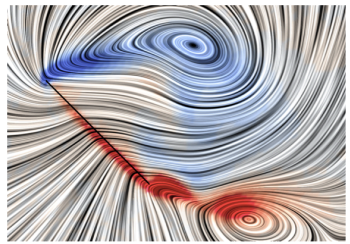

o) $s / c=8$ (CFD)

Figure 11: Comparison of slow surge flow fields from AFRL dye flow visualisation in the left hand column, UMD/AFRL PIV data showing vorticity field with vectors superimposed in the second column and RVC CFD, vorticity field with vectors superimposed and LIC in the third and fourth columns respectively. 
estimates provide two inputs required for the model in the work by Babinsky et al. [13] as outlined in Section B. The vortex trajectories from experimental data described herein have all been extracted following the Graftieaux et al. [21] non-local vortex detection scheme ${ }^{e}$. The RVC, CFD data trajectories were found by identifying in-plane critical points given by zero-crossing points of the velocity profiles in the $x$ and $z$ directions ${ }^{\mathrm{f}}$.

Figure 12 shows schematically how $x_{L E V}$ and $x_{T E V}$ are defined in space relative to the $\mathrm{LE}$ and TE of the wing respectively. Variations in the relative $z$-position of the LEV and TEV will likely only affect forces in the drag direction $[23,24]$, and hence are not discussed here.

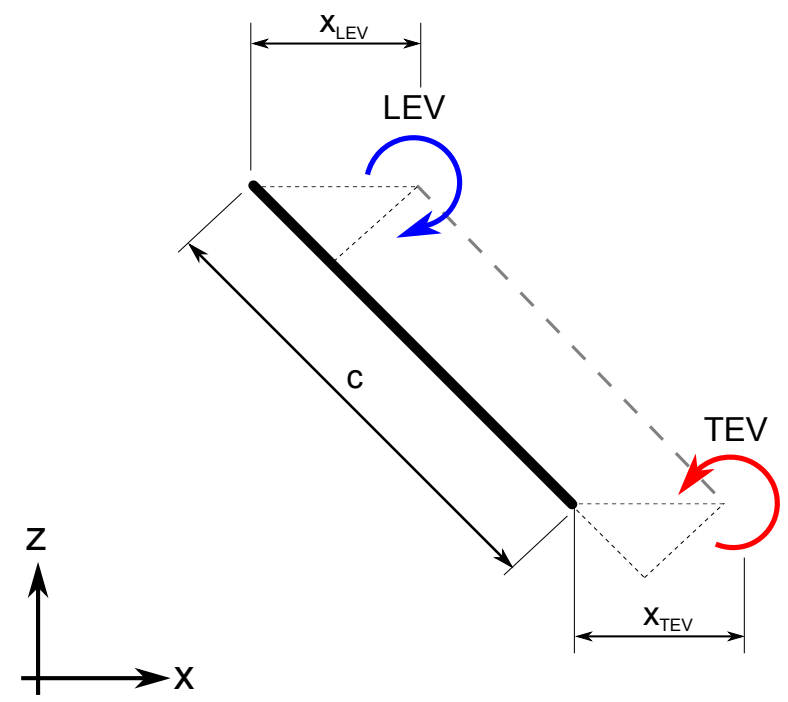

Figure 12: LEV and TEV position definition.

LEV trajectories are compared in Figure 13, where the LEV $x$-position is non - dimensionalised by the wing chord and plotted against advective time, $s / c$. TEV trajectories from the Cambridge group alone, for the fast and slow surge cases are compared in Figure 14. No other TEV trajectory data was available. The LEV data of Figure 13 shows remarkable agreement in the range, $0<s / c<2$ not only between the two different experimental facilities and CFD data but also between the fast and slow cases. Similarly to the LEV data, there is a striking correlation between the TEV trajectories for the fast and slow cases seen in Figure 14. It is noted that for both the LEV and TEV datasets, at values of greater than

${ }^{\mathrm{e}}$ The method of Graftieaux et al. [21] as implemented here involves computing the centroid of a vortex bounding core, $\gamma_{2}$. The advantage of using this method with PIV data is that the flowfield doesn't need to be differentiated and hence there is no amplification of any experimental noise. A $\gamma_{2}$ threshold of $2 / \pi$ is used

${ }^{\mathrm{f}} \mathrm{A}$ critical point exists when both the $x$ and $y$-components of velocity are equal to zero. This critical point was automatically classified into different types (i.e. foci, saddles) as in Phillips et al. [22] and the foci were taken to be the central axis of the vortex. 
$s / c \approx 2$, a lack of LEV/TEV coherency sometimes makes detection problematic and data becomes noisy and unreliable. The UMD/AFRL LEV data for the fast surge case is the notable exception to this, with data available until $s / c \approx 3.5$. Despite a lack of data at longer advective timescales, it is expected that the LEV and TEV will eventually drift at the steady state velocity ${ }^{\mathrm{g}}$, but it is also recognised that new LEVs/TEVs may form as well.

All the datasets shown in Figure 13 show an approximately linear increase in LEV core distance relative to the LE with increasing advective time which suggests that the LEV moves away from the wing at a relatively constant velocity (at least up until approximately $s / c=2$ ). Furthermore, there is good agreement in general between $\operatorname{Re}=10,000$ and $\operatorname{Re}=$ 20,000 hence there is not much of a Reynolds number effect within this limited range.

Similar to the LEV, the TEV data shows a linear increase in TEV core position relative to the TE with increasing advective time. When comparing with the LEV data however, it is noticed that the gradient of TEV advection is much steeper (approximately 2.5 times greater), as the TEV advects aft faster than the TEV.

A crude approximation of the relative separation between the LEV and TEV at the start of the surging motion may be estimated from Figures 13 and 14, where both datasets have a best fit line beginning at the origin, yielding a value for core $x$-separation (at $\alpha=45^{\circ}$ ) of approximately $0.7 x / c$, which corresponds to approximately $1 / \sqrt{2}$ or $c \cdot \cos \alpha$, where $\alpha=45^{\circ}$ here. The relative LEV/TEV core initial horizontal separation can thus be approximated as $\left(x_{L E V}-x_{T E V}\right) \approx c \cdot \cos \alpha$.

A first approximation of the relative advection velocities is made through comparison of the approximate gradients of the data of Figures 13 and 14, yielding $a b s\left(u_{L E V}-u_{T E V}\right) \approx$ $0.5 \cdot U_{\infty}{ }^{\mathrm{h}}$

\section{LEV Circulation}

The final input for the low-order model (see again Section I. B) is the LEV circulation. A contour integral approach has been used to estimate the circulation from PIV data by Pitt Ford [25]. Circulation was computed from CFD for the slow surge case by the RVC group. Figure 15 shows circulation data non-dimensionalised by the theoretical circulation, $\Gamma_{\infty}$ against advective time ${ }^{i}$. The classic Wagner curve [26], which predicts the delay in attainment of steady state circulation for an impulsively started flat plate wing acts as a reference. The fast surge case in Figure 15, shows the closest match to the theoretical prediction by Wagner, however in general despite the trend of the circulation following

\footnotetext{
gThis assumes that there is no viscous dissipation of the vortices beforehand, which we know is also occurring.

${ }^{\mathrm{h}}$ Note that the advection velocities are scaled here relative to the freestream velocity of the wing in the translation part of the motion.

${ }^{\mathrm{i}}$ The theoretical circulation here is invoked via Kutta-Joukowski as $\Gamma_{\infty}=\pi \alpha U_{\infty} \mathcal{C}$, which is equivalent to $C_{L}=2 \pi \alpha$.
} 


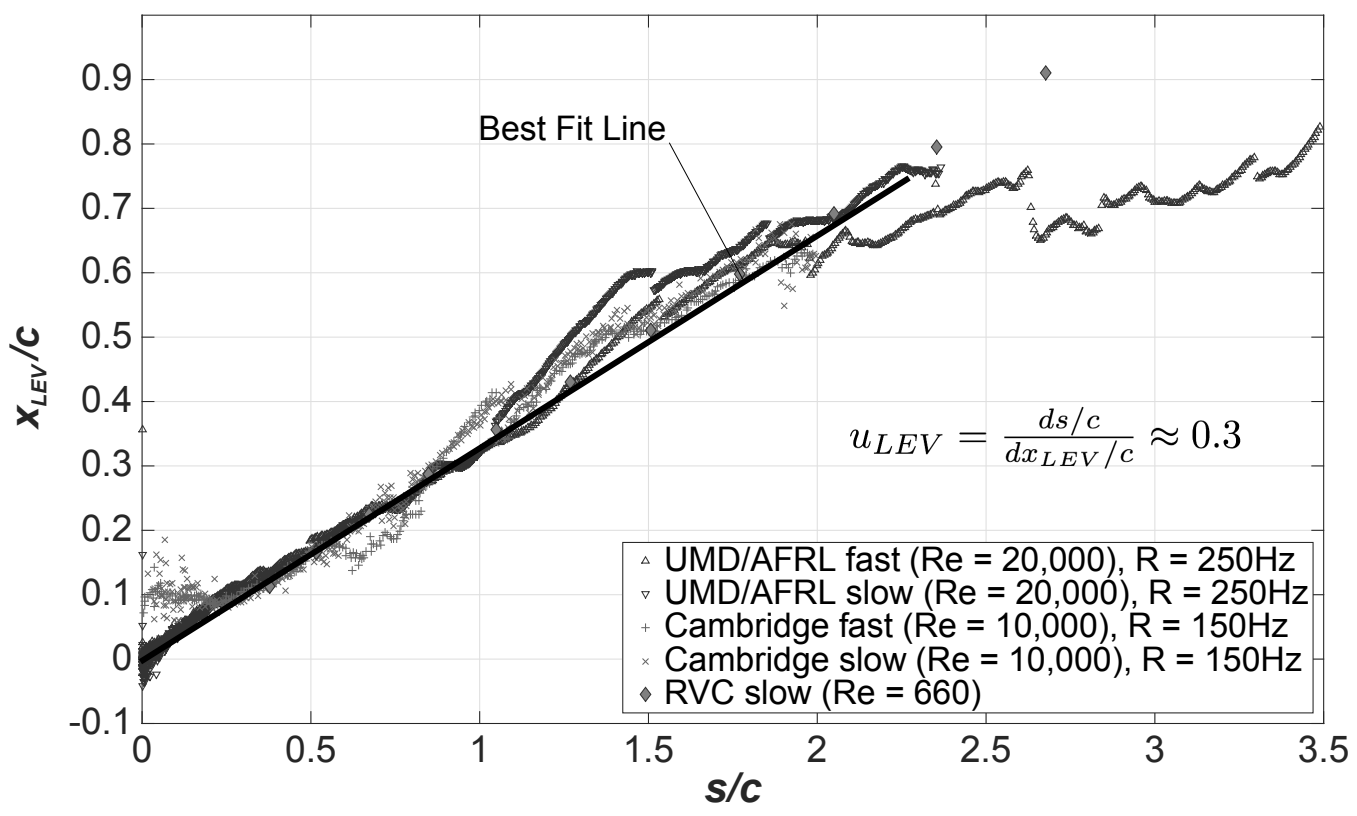

Figure 13: LEV trajectory comparison.

that predicted, the absolute values deviate somewhat. There is a reasonable agreement in trend between the PIV and CFD data for the slow surge case, despite differences in Reynolds number. The low-order model proposed by Babinsky et al. [13] discusses how the theoretical Wagner curve may be modified to provide a more realistic prediction.

\section{E. Variations on the Canonical Case}

Here we briefly discuss some variations on the canonical AVT-202 case.

\section{Effect of Reynolds Number on Forces}

Some of the available force and vortex trajectory data was conducted at different Reynolds numbers. It has already been demonstrated that LEV trajectories from as low as $\operatorname{Re}=660$ show good agreement with the canonical case. To further check if Reynolds number independence can be assumed, an experimental Reynolds number study was conducted at the Cambridge tow tank facility and a computational study was conducted by Gozukara at Aselsan.

Figure 16 (a) compares the Cambridge force history of an $R=8$ wing at $\operatorname{Re}=10,000$ with an $R=4$ and $R=8$ wing at $\operatorname{Re}=20,000$ for a surge over 1 c. A collapse of the curves is observed albeit with some subtle differences in the magnitude of the initial spike in lift. This suggests that Re effects appear to be small between $\operatorname{Re}=10,000$ and $\operatorname{Re}=20,000$ for the Cambridge facility. 


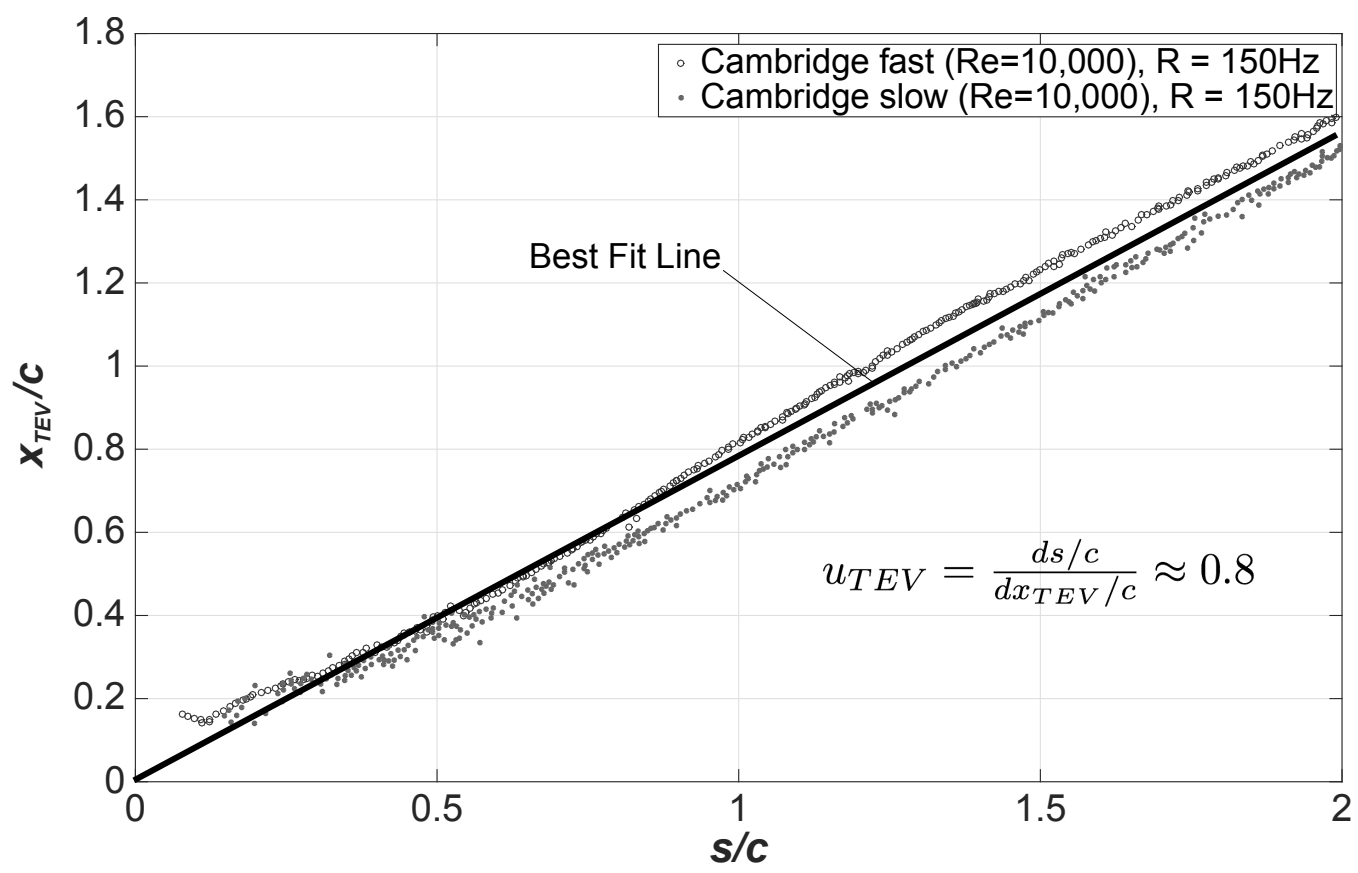

Figure 14: TEV trajectories determined from PIV datasets.

Figure $16 \mathrm{~b}$ compares the Aselsan force history of an $R=4$ wing at Reynolds numbers of 3,600 and 10,000 for a surge over 1c. Once more there is a collapse of the curves observed. The one noticeable discrepancy is the magnitude of the second lift maximum, which is approximately $6 \%$ greater for $\operatorname{Re}=3,600$, with respect to $\operatorname{Re}=10,000$. Again the CFD data suggests that Re effects appear to be small within this limited range.

\section{Aspect Ratio Variations}

Here the canonical-case kinematics are retained, but the plate $R$ is variedi. Figure 17 compares $R=2,4$ and 8 . There is a generally good correlation in trend between all datasets. The $R=8$ lift history (Stevens) does not vary greatly from the $R=4$ canonical case datasets. The $R=2$ (Manar) data appears to have a lower asymptotic value of lift at longer advective times, once more calling into question the true steady state lift value.

For the fast surge case, the secondary maximum in lift at around $s / c=7.5$, which was previously discussed for the canonical cases is not visible in the $R=2$ case whereas it is especially strong in the $R=8$ case. The $R=2$ wing is likely to have a more threedimensional flow on account of free-end effects and this might affect the organisation of the flowfield. It is recalled that the canonical case data of Stevens doesn't show a secondary maximum in lift and this could be linked to the use of a physical $R=2$ wing with a skim

IIt is useful to distinguish between physical and effective AR. The physical $R$ is the full span wing of that $R$, whereas the effective $R$ stems from an image (symmetry) plane; the ramifications of this are explored. 


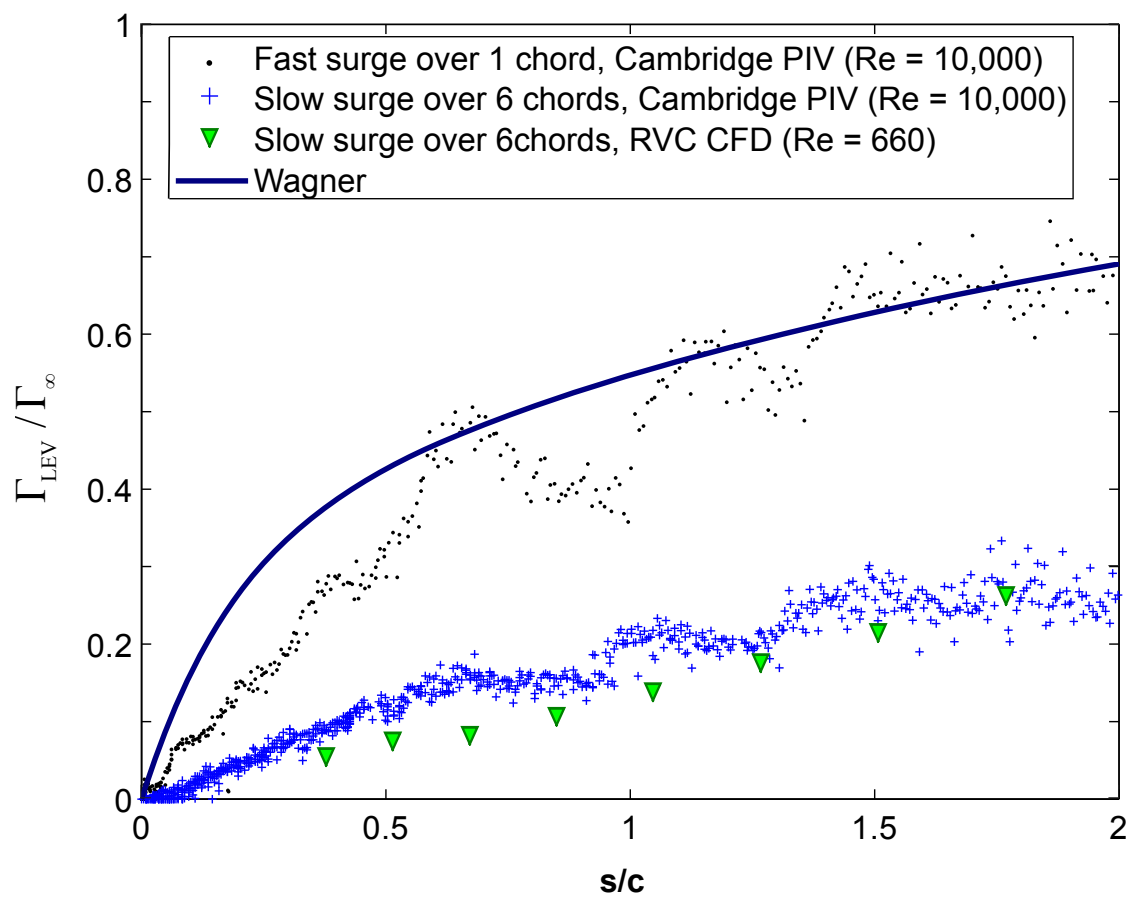

Figure 15: Normalised LEV circulation histories for both fast and slow surge cases, calculated from PIV and CFD data compared with Wagner's theoretical curve.

plate to create an effective $R=4$ wing, which in reality may not fully suppress end effects at the skim plate end.

The slow surge $R$ variation lift history is shown in Figure 18 . There is very good agreement between all datasets, while surging from $s / c=0$ to $s / c \approx 5$. After $s / c \approx 5$, where the flow might be considered fully stalled, there seems to be a greater discrepancy in the lift history.

There seems to be some noticeable $R$ effect in the Cambridge slow surge data, with the effective $R=8$ wing producing a higher lift than the corresponding effective $R=4$. The $R=2$ data of Manar is interestingly very similar to the effective $R=4$ (physical $R$ $=2$ ) data of Stevens. This again hints that there is an effect of flow three-dimensionality on the lift history and that the use of a symmetry plane may affect the force history. 


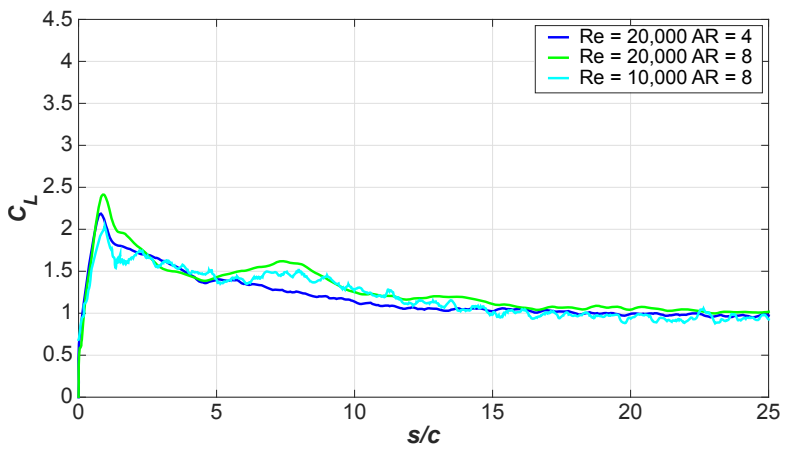

a) Cambridge experimental data.

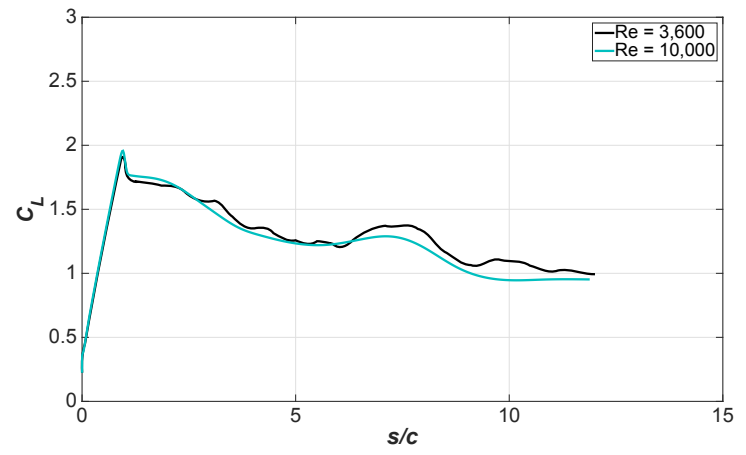

b) Aselsan computational data.

Figure 16: Reynolds number effect on force history comparison for fast surge (1c) case

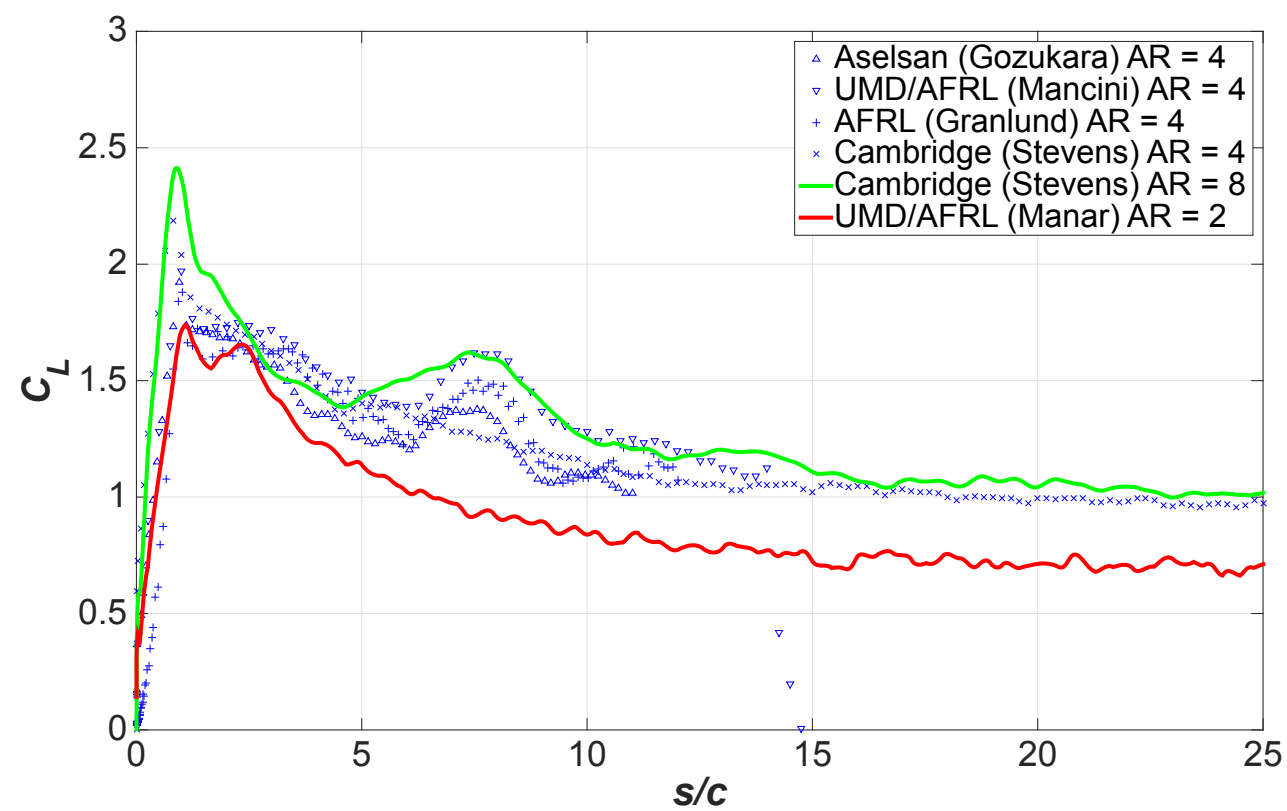

Figure 17: Fast case $R$ variation. 


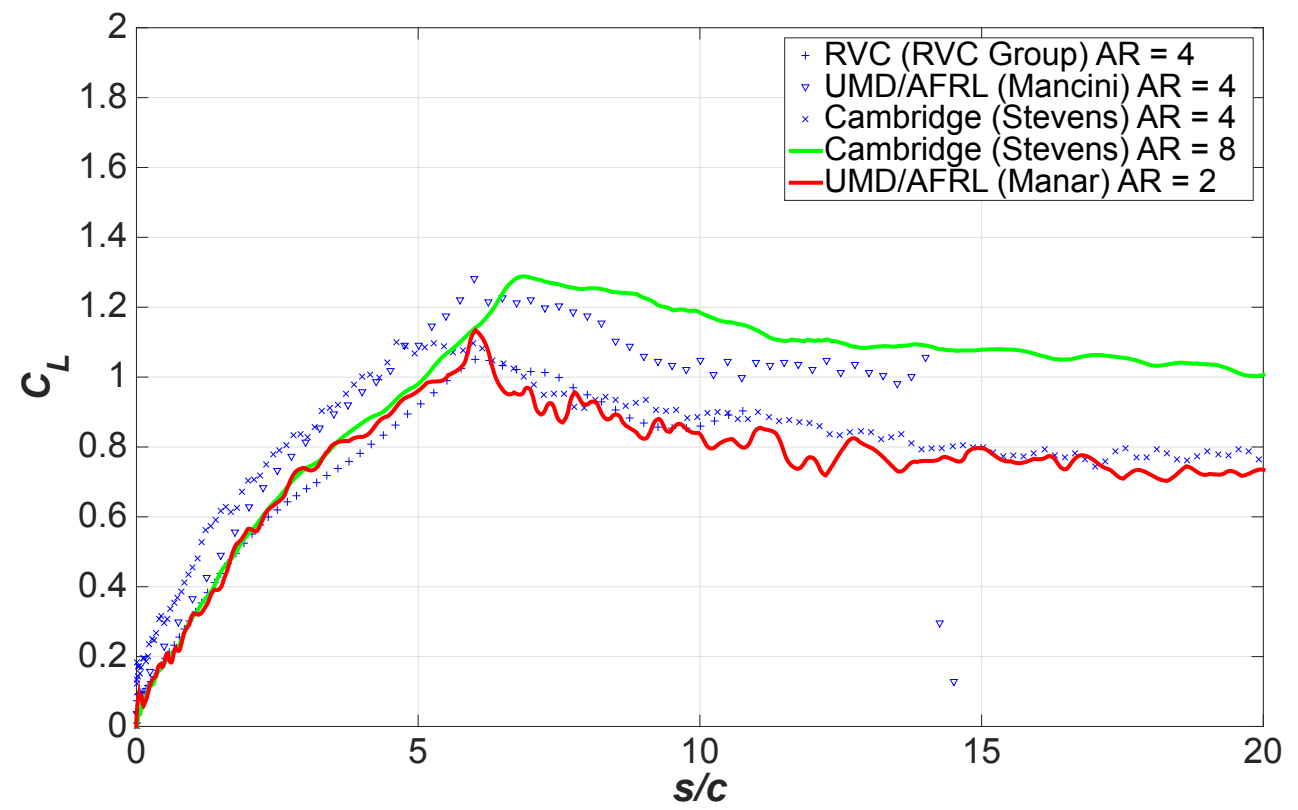

Figure 18: Slow case $A$ variation. 


\section{Conclusions}

This paper has shown the results of a concerted effort from the AVT-202 task group to gain a deeper understanding of high incidence surging flat plate wings at low Reynolds numbers. A striking correlation of lift history data and flow topology from both experimental and computational datasets has been demonstrated. Further, some key flow topology has been linked with anomalous features in the force history such as the second lift maximum around $s / c=7.5$ in the fast surge case, which correlates to a pseudo-reattachment of the flow.

Several important parameters have been estimated that may act as inputs for the loworder model described in the companion work by Babinsky et al. [13]. These may be summarised as follows:

1. The LEV/TEV core separation distance at the beginning of surging, $\left(x_{L E V}-x_{T E V}\right)$, is approximated by $c \cdot \cos \alpha$.

2. The LEV/TEV, relative advection velocity, $\left(u_{L E V}-u_{T E V}\right)$ is approximated as $0.5 \cdot U_{\infty}$.

3. The circulation of the LEV, $\Gamma_{L E V}$, approximately follows the general trend of Wagner for the fast surge case. However, a modification to the Wagner function, which takes the kinematics into account may prove to be more realistic.

Some variations of the canonical case have also been briefly examined in this paper. It was found that Reynolds number effects appear to be small in the range $\operatorname{Re}=660$ to $\operatorname{Re}=$ 20,000 for both experimental and computational datasets. Additionally it was found that $R$ variations don't alter the general trend of forces, however there are some effects such as the absence of a secondary lift maximum in the fast surge case and a lower asymptotic (steady state) lift coefficient in both the fast and slow surge case, when an $R=2$ wing is used. This $R$ effect may be caused by flow three-dimensionality, which is likely to have a strong influence for small $R$ wings.

Future work involves expansion of the kinematic envelope and further study on the vortex advection mechanics and circulation.

\section{Acknowledgements}

Dr. Charlie Pitt Ford is gratefully acknowledged for providing some data, which has been analysed in this body of work. R.J. Bomphrey and N. Phillips were supported by the Engineering and Physical Sciences Research Council (EP/H004025/1 to RJB). R.J. Bomphrey and T. Nakata were supported by the Biotechnology and Biological Sciences Research Council (BB/J001244/1 to RJB). 


\section{References}

[1] McMichael and Francis, "Micro Air Vehicles - Towards a New Dimension in Flight," Tech. rep., USAF, DARPA TTO document, 1997.

[2] Shyy, W., Lian, Y., Tang, J., Viieru, D., and Liu, H., Aerodynamics of Low Reynolds Number Flyers, Cambridge University Press, 2008.

[3] Lentink, D. and Dickinson, M. H., "Biofluiddynamic Scaling of Flapping, Spinning and Translating Fins and Wings," Journal of Experimental Biology, Vol. 212, 2009, pp. 2691-2704.

[4] AVT-149, T. G., "Unsteady Aerodynamics for Micro Air Vehicles,” Tech. rep., NATO RTO, 2010.

[5] Dickinson, M. H. and Götz, K. G., "Unsteady Aerodynamic Performance of Model Wings at Low Reynolds Numbers," J. exp. Biol., Vol. 174, 1993, pp. 45-64.

[6] Beckwith, R. M. H. and Babinsky, H., "Impulsively Started Flat Plate Flow," Journal of Aircraft, Vol. 46, No. 6, Engineering Notes, November-December 2009, pp. 2186-2188.

[7] Ringuette, M. J., Milano, M., and Gharib, M., "Role of the tip vortex in the force generation of low-aspect-ratio normal flat plates," J. Fluid Mech, Vol. 581, 2007, pp. 453-468.

[8] Batchelor, G. K., An Introduction to Fluid Dynamics, Cambridge University Press, 1967.

[9] Chen, K. K., Colonius, T., and Taira, K., “The leading-edge vortex and quasisteady vortex shedding on an accelerating plate." Physics of Fluids, Vol. 22, 2010.

[10] Baik, Y. S., Bernal, L. P., Granlund, K., and Ol, M. V., "Unsteady force generation and vortex dynamics of pitching and plunging aerofoils," J. Fluid Mech, Vol. 709, 2012, pp. 37-68.

[11] Bansmer, S. E. and Radespiel, R., "Flapping Flight: High Thrust and Propusive Efficiency due to Forward Gliding Oscillations," AIAA Journal, Vol. 50, No. 12, 2012, pp. 2937-2942.

[12] “Extension of Fundamental Flow Physics to Practical MAV Aerodynamics (AVT-202)," Tech. rep., NATO, STO, 2016.

[13] Babinsky, H., Stevens, P. R. R. J., Jones, A. R., Bernal, L. P., and Ol, M. V., “Low Order Modelling of Lift Forces for Unsteady Pitching and Surging Wings," AIAA SciTech 2016, 2016.

[14] Morgan, C., Unsteady Vortex Interactions Related to a Formula One Car Front Wing and Wheel, Ph.D. thesis, University of Cambridge, 2010.

[15] Granlund, K., Ol, M. V., and Bernal, L., "Experiments on Pitching Plates: Force and Flowfield Measurements at Low Reynolds Numbers," 49th AIAA Aerospace Sciences Meeting, AIAA, 2011.

[16] Liu, H., "Integrated modelling of insect flight: From morphology, kinematics to aerodynamics," Journal of Computational Physics, Vol. 228, No. 2, February 2009, pp. 439-459.

[17] Eldredge, J. D. and Wang, C., "A Computational Study of a Canonical Pitch-up, Pitch-down Wing Maneuver," 39th AIAA Fluid Dynamics Conference, San Antonio Texas, June 2009.

[18] Wang, C. and Eldredge, J. D., "Low-order phenomenological modeling of leading-edge vortex formation," Theor. Comput. Fluid Dyn., Vol. 27, 2012, pp. 577-598. 
[19] Son, O., Cetiner, O., Stevens, P. R. R. J., Babinsky, H., Manar, F. H., Mancini, P., Jones, A. R., Ol, M. V., and Gozukara, A. C., "Parametric variations in aspect ratio, leading edge and planform shapes for the rectilinear cases of AVT-202," AIAA SciTech 2016, 2016.

[20] Cabral, B. and Leedom, L. C., "Imaging Vector Fields Using Line Integral Convolution," Assocication for Computing Machinery, Vol. ACM-0-89791-601, 1993, pp. 263-270.

[21] Graftieaux, L., Michard, M., and Grosjean, N., "Combining PIV, POD and Vortex Identification Algorithms for the Study of Unsteady Turbulent Swirling Flows," Meas. Sci. Technol., Vol. 12, 2001, pp. 1422-1429.

[22] Phillips, N., Knowles, K., and Bomphrey, R., "The effect of aspect ratio on the leading-edge vortex over an insect-like flapping wing." Bioinspiration and Biomimetics, Vol. 10, No. 5, October 2015.

[23] Kármán, T. V. and Sears, W. R., “Airfoil Theory for Non-Uniform Motion," Journal of the Aeronautical Sciences, Vol. 5, No. 10, 1938, pp. 379-390.

[24] PittFord, C. W., Unsteady Aerodynamic Forces on Accelerating Wings at Low Reynolds Numbers, Ph.D. thesis, University of Cambridge, 2013.

[25] Pitt Ford, C. W. and Babinsky, H., "Lift and the leading-edge vortex," J. Fluid Mech, Vol. 720, 2013, pp. 280-313.

[26] Wagner, H., "Über die Entstehung des Dynamischen Auftriebes von Tragflügeln," Zeitschrift für Angewandte Mathematik und Mechanik, Vol. 5, 1925, pp. 17-35. 